\title{
Patrón de consumo de verduras en una población infantil de Pamplona: Estudio Cualitativo
}

\author{
Consumption pattern of vegetables in a child population of Pamplona: Qualitative study \\ Irene Botía-Rodríguez' orcid.org/0000-0001-9862-3115 \\ Gabriel Antonio Cardona-Arguello1 orcid.org/0000-0001-6738-6348 \\ Lennys Carvajal-Suárez ${ }^{1 *}$ orcid.org/0000-0001-6490-7670
}

1 Facultad de Salud, Universidad de Pamplona, Pamplona, Colombia

Fecha de recepción: Abril 13 - 2019

Fecha de revisión: Septiembre 24-2019

Fecha de aceptación: Diciembre 19 - 2019

Botía-Rodríguez I, Cardona-Arguello G, Carvajal-Suárez L. Patrón de consumo de verduras en una población infantil de Pamplona: Estudio Cualitativo. Univ. Salud. 2020;22(1):84-90. DOI: https://doi.org/10.22267/rus.202201.178

\section{Resumen}

Introducción: La alimentación adecuada es fundamental para el desarrollo físico e intelectual pleno de los niños. Objetivo: Analizar el patrón de consumo de verduras en una población infantil de Pamplona, Colombia. Materiales y métodos: Se realizó una investigación cualitativa mediante la metodología de discusión grupo focal con la participación de madres de familia de un hogar infantil. La información fue analizada a través de la propuesta de Attride-Stirling. Resultados: Las participantes consideraron que el consumo de verduras es importante e influye positivamente en el estado de salud. El consumo es afectado por la baja cantidad y poca frecuencia de suministro, preferencias limitadas por algunas preparaciones, ejemplo inadecuado en padres de familia. La frecuencia de compra, el lugar, precio y características organolépticas, son factores que condicionan la compra de verduras en el hogar. Se mencionaron estrategias positivas y negativas para incentivar la ingesta en los niños. Conclusiones: Las madres tienen conocimientos asertivos sobre el consumo de verduras y su relación con la salud en los niños. La cantidad consumida es inadecuada puesto que difiere de las recomendaciones establecidas. Los elogios, animación y compañía de padres para incentivar su consumo son estrategias positivas, aunque utilizan el castigo y el premio.

Palabras clave: Verduras; patrones alimentarios; alimentación; nutrición en salud pública; salud (Fuente: DeCS, Bireme).

\begin{abstract}
Introduction: Adequate nutrition is fundamental for the physical and intellectual development of children. Objective: To analyze the vegetable consumption pattern of a child population in Pamplona (Colombia). Materials and methods: We conducted a qualitative research with mothers that used children's day care facilities applying the focus group discussion methodology. The information was analyzed with the Attride-Stirling tool. Results: Participating mothers considered that vegetable consumption is important and has a positive effect on children's health. A poor consumption is caused by low quantity and frequency of supply, limited preferences for some food preparations, and inadequate examples set by parents. The purchasing frequency, place, price and organoleptic characteristics of vegetables are important factors that limit their purchase at home. Some positive and negative strategies are suggested to encourage the ingestion of vegetables in children. Conclusions: Mothers have assertive knowledge about vegetable consumption and its relation to children's health. The level of vegetable consumption was low as it differs from the established recommendations. Praise, motivation and companionship of parents are positive strategies to encourage vegetable consumption, although some prefer punishment and reward.
\end{abstract}

Key words: Vegetables; feeding behavior; feeding; nutrition public health; health (Source: DeCS, Bireme).

\footnotetext{
*Autor de correspondencia

Lennys Carvajal Suárez

e-mail: lennys.carvajal@unipamplona.edu.co
} 


\section{Introducción}

Según la Organización Mundial de la Salud (OMS), la insuficiente ingesta de frutas y verduras hace parte de los 10 factores de riesgo principales que contribuyen a la mortalidad. Si hubiera un consumo mundial suficiente se contribuiría a la prevención de enfermedades no transmisibles, como las cardiovasculares y algunos cánceres(1).

Se ha evidenciado que existe una estrecha relación entre los hábitos alimentarios y la salud de una población, a medida que se deja de llevar una alimentación saludable, existe un aumento de enfermedades crónicas como la obesidad, diabetes mellitus, enfermedades cardiovasculares, cáncer y otras patologías, que pueden ocasionar mayor morbilidad y mortalidad, constituyéndose en un problema de salud pública.

En la Encuesta Nacional de la Situación Nutricional (ENSIN 2005), se identificaron algunas problemáticas relacionadas con el consumo de alimentos; más de la tercera parte de la población colombiana presentó bajo consumo de frutas $y$ verduras $y$ altas prevalencias de deficiencias en la ingesta de micronutrientes(2). La ENSIN 2010, reportó que el $64,9 \%$ de los colombianos y el 10,3\% de la población infantil consumen verduras cocidas(3).

La buena alimentación es fundamental en los primeros años de vida para que los niños y las niñas tengan más oportunidad de vivir sanos y que el desarrollo físico e intelectual sea pleno. Una deficiente ingesta durante la primera infancia tiene repercusiones irreversibles en el individuo y la sociedad en general, tales como vulnerabilidad a enfermedades y bajo desempeño educativo(4). En el estudio realizado por Alderete et al.(5) se resalta que se ha producido una modificación de los hábitos alimentarios principalmente debido al aumento de la producción de alimentos procesados, la rápida urbanización y el cambio en los estilos de vida; existe un consumo elevado de alimentos hipercalóricos, grasas saturadas, grasas trans, azúcares simples y sodio; sumado a una ingesta insuficiente de frutas, verduras y fibra dietética, dado específicamente por gustos de los padres que son adquiridos por sus hijos, falta de costumbre de consumo habitual, de variedad en la preparación de comidas que incluyan verduras como ingrediente principal, y de conocimiento, creatividad, y paciencia. Adicionalmente, Girón et $a l .(6)$ destacan que un factor que influye en los hábitos alimentarios de las familias es la forma de vida por tradiciones, es así como a pesar de tener al alcance productos oriundos de la zona de alto valor nutritivo como frutas y verduras no las incluyen en su dieta diaria. Así pues, cuantificar y detallar con precisión el consumo de alimentos en ocasiones es una tarea difícil, pues los diferentes tipos de encuestas para describir un determinado patrón alimentario son herramientas con características particulares, cada una con sus ventajas, pero también con ciertos inconvenientes(7). Por ello, se ha identificado la necesidad de disponer de métodos cualitativos que son útiles para la investigación, exploración e indagación desde la subjetividad de los participantes, sobre diversos aspectos que inciden notablemente en el patrón de consumo de alimentos, además de los criterios para la decisión, selección, preparación, compra y el consumo de estos. Dentro de ellos se destaca la discusión de grupo focal, técnica en la cual se obtienen datos a partir de reuniones en grupos con personas que representan el objeto de estudio, siendo una de las más usadas en los estudios cualitativos al involucrar la observación e interacción con los entrevistados ${ }^{(8,9)}$. Una de sus mayores ventajas es que se basa en la tendencia humana de formar opiniones y actitudes durante la interacción entre los individuos, que no se logra con cuestionarios cerrados o entrevistas individuales porque a cada individuo se le permite emitir opiniones(8).

Por tanto, ésta investigación tiene por objeto identificar los factores asociados al consumo de este grupo de alimentos en los beneficiarios del Hogar Infantil Niño Jesús de Praga, institución sin ánimo de lucro adscrito al Instituto Colombiano de Bienestar Familiar (ICBF) que brinda atención integral a niños y niñas entre 18 meses y 5 años de edad.

\section{Materiales y métodos}

Se realizó una investigación cualitativa que incluye el desarrollo de la metodología de discusión grupo focal. En su desarrollo se contemplaron las siguientes etapas: Reconocimiento del tema de discusión, identificación y selección de los participantes, moderadora y secretaria, diseño de la guía de discusión temática, preparación logística, organización de material didáctico y desarrollo del taller que incluye las siguientes fases: Inducción, conducción, discusión y clausura. La selección de los participantes se realizó mediante muestreo intencional, el cual hace referencia a un tipo de 
individuos considerados representativos de la estructura social ya que son informantes clave(10). Se consideraron como requisitos mínimos, personas mayores de 18 años, responsables de la compra y preparación de los alimentos en el hogar, lo cual facilita el desarrollo de la guía temática.

El grupo focal se realizó en un aula del hogar infantil con 12 madres que aceptaron participar en el estudio, el cual fue dirigido por dos nutricionistas dietistas en formación de la Universidad de Pamplona para desempeñar el rol de moderadora y secretaria respectivamente. Fue elaborada una guía temática con el fin de dirigir la discusión, para lo cual se abordaron preguntas referentes a conocimientos, compra, precio, frecuencia de consumo y estrategias utilizadas para motivar el consumo de verduras en la población infantil.

El análisis del material obtenido se efectúo con base en la propuesta de Attride-Stirling denominada redes temáticas, a partir de la cual se organizan los datos cualitativos de acuerdo a los temas que surgen en diferentes niveles, que incluye seis pasos básicos: 1) Codificación del material: reducción del material y ubicación de los segmentos textuales con significado relevante para el análisis, 2) Identificación de los temas: se destacan los contenidos de interés y se trasladan a un nuevo documento, 3) Construcción de redes temáticas: identificación de grandes grupos temáticos codificados con nombre y agrupados por temas semejantes, 4) Descripción y exploración de las redes temáticas: constituye la primera parte del análisis, teniendo en cuenta que en la construcción de las redes se deben realizar varias lecturas para mejorar la comprensión y el significado de los temas, 5) Resumen de los temas: elaboración de una síntesis con los temas principales y características que lo componen, 6) Interpretación de las características

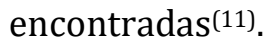

\section{Consideraciones éticas}

La investigación se consideró sin riesgo de acuerdo a lo estipulado en la Resolución No 8430 del 04 de octubre de 1993 propuesta por el Ministerio de Salud de Colombia, en la cual se establecen las normas científicas, técnicas y administrativas para la investigación en salud(12). Se obtuvo el consentimiento institucional avalado por la Asociación de Padres de Familia del Hogar Infantil, y de las madres de familia participantes a través de un instrumento previamente diseñado.

\section{Resultados}

El grupo focal fue grabado en su totalidad con una duración de 1 hora y 30 minutos, transcrito en 24 páginas. Posterior a la codificación del material se obtuvo 7 páginas con los segmentos de texto que tenían significado relevante para el análisis, los fragmentos fueron agrupados en 4 redes temáticas: Conocimiento del consumo de verduras, consumo de verduras en la población infantil, factores condicionantes para la adquisición de verduras y estrategias utilizadas para incentivar el consumo de verduras en los niños y niñas (Tabla 1).

En la red temática denominada "conocimiento del consumo de verduras", se recopilaron dos temas: Importancia y relación del consumo de verduras con el estado de salud y beneficios del consumo de verduras. Al respecto, las madres consideraron importante la ingesta de estos alimentos debido a que aportan vitaminas y minerales que favorecen la salud

Tabla 1. Redes temáticas sobre percepción de las familias frente a compra y consumo de verduras en niños del Hogar Infantil Niño Jesús de Praga, Pamplona

\begin{tabular}{|c|c|}
\hline Redes temáticas & Temas \\
\hline R1 Conocimiento del consumo de verduras & $\begin{array}{l}\text { Importancia y relación del consumo de verduras y el estado de salud } \\
\text { Beneficios del consumo de verduras }\end{array}$ \\
\hline \multirow[t]{5}{*}{ R2 Consumo de verduras en la población infantil } & Frecuencia del consumo en el hogar \\
\hline & Tiempos de comida en que son ofrecidas \\
\hline & Presentación de las verduras \\
\hline & Cantidad de verduras consumidas \\
\hline & Factores que afectan el consumo \\
\hline \multirow[t]{4}{*}{ R3 Factores condicionantes para la adquisición de verduras } & Frecuencia de compra \\
\hline & Lugar de compra \\
\hline & Precio \\
\hline & Características al momento de comprar \\
\hline \multirow{3}{*}{$\begin{array}{l}\text { R4 Estrategias utilizadas para incentivar el consumo de } \\
\text { verduras en los niños y niñas }\end{array}$} & Castigo \\
\hline & Ejemplo \\
\hline & Motivación \\
\hline
\end{tabular}


de los niños, refiriendo expresiones como: "Yo diría que, el consumo de verduras para el buen crecimiento de los niños (...)"; "El aporte de vitaminas que traen las verduras (...)"; "Una dieta saludable, para la buena salud (...)".

Con respecto a la relación del consumo de verduras y el estado de salud y nutrición de los niños(as), las madres refirieron que éste grupo de alimentos influye positivamente en la salud, manifestando las siguientes opiniones: "Aportan mucho hierro, vitaminas, (...)"; "Aportan vitaminas, minerales y fibra; "Ayudan al organismo a trabajar adecuadamente permitiendo que los niños crezcan sanos y fuertes (...); además, lo relacionan con el "estado anímico y el suministro de energía (...)”.

En cuanto a la segunda red temática denominada "consumo de verduras en la población infantil", se resaltó el consumo de verduras propias de la región como zanahoria, tomate, habichuela, arveja, brócoli, lechuga, repollo, entre otras, las cuales son ofrecidas principalmente al almuerzo y la cena en preparaciones como: sopas, pericos, mezcladas con arroz, guisos, zumos, purés o tortas. Referente a la cantidad suministrada, las participantes refirieron las siguientes equivalencias: dos cucharadas soperas para ensaladas, si son rodajas de tomate tres unidades, en preparaciones como guiso o pericos de medio a un pocillo tintero y si son zumos se ofrece un vaso. La frecuencia de consumo de verduras en este grupo poblacional es de 2 a 3 veces por semana.

Entre los factores que afectan el consumo de verduras en los niños(as), se destacó la poca frecuencia de consumo en el hogar, las conductas negativas por otros niños, manifestando expresiones como: "Por lo menos mis chiquitines estaban acostumbrados a comer verduras y ahora briego para que se coman la zanahoria y la habichuela, empiezan a decir guácala, eso es una imitación que debió aprenderla de alguien (...)". Otro factor asociado al consumo de verduras es la inclusión de preparaciones diferentes a las que se ofrecen en el hogar familiar, "Porque ellos están acostumbrados a un tipo de preparación y tienen un cambio que a ellos de pronto no les gusta a como lo están consumiendo, pues ya uno vuelve hacerlo y ellos ya no lo van a consumir (...)"

En relación a los factores condicionantes para la adquisición de las verduras; se identificó que algunas madres realizan diariamente la compra, otras cada 3,
4 u 8 días de acuerdo a las necesidades, resaltando las siguientes opiniones: "Una cantidad moderada le puede durar hasta ocho días, cuatro días según (...)"; "Cada ocho días más o menos (...)"; "De acuerdo a la necesidad (...)".

Los lugares de preferencia referidos para la compra de verduras correspondieron al mercado principal de la ciudad ya sea afuera o dentro de él, centro de acopio, tiendas, fincas o huerta casera. Los mejores precios ofertados se encuentran en el mercado, centro de acopio y mercados campesinos. Las características priorizadas por las madres al momento de comprar las verduras fueron "que se vean fresquitas (...)", "tamaño (...)", "firmeza (...)", "el estado (...)" y el color, "El brócoli cuando está fresco tiene un color verde, y si no tiene un color amarillo (...)".

Acerca de las estrategias utilizadas para incentivar el consumo de verduras en los niños(as), las madres refirieron decoración de los platos, barras, caritas felices, consumo en compañía de los padres, además de juegos "El avioncito", "Yo me como una y usted se come otra" (...)", premios "le damos un dulce", competencias "El que termina primero gana", o castigo "La correa" (...).

\section{Discusión}

La familia es la base fundamental de la sociedad, y los padres son el primer ejemplo de los hijos(13). El resultado obtenido en este estudio sobre la importancia del consumo de verduras coincide con los resultados referidos por Gamarra et al. donde se evidencia que las madres perciben que el consumo diario de frutas y verduras es una práctica adecuada por contener vitaminas y evitar el estreñimiento(14).

Las verduras son fuente importante de vitaminas y minerales, que contribuyen a la regulación de diferentes procesos vitales(15). Según la OMS, los beneficios de las frutas y verduras para la salud, no pueden atribuirse a los efectos de un solo nutriente $o$ de una mezcla particular de nutrientes, sino a todo un cúmulo de beneficios que se obtienen cuando este grupo de alimentos se consume en cantidades y variedades suficientes. El consumo adecuado de frutas y verduras, armonizado con otros hábitos alimentarios saludables es un factor fundamental para prevenir y reducir las deficiencias de micronutrientes que afectan a la población colombiana, máxime si dicho consumo es promovido desde las primeras etapas de la vida. Este consumo 
además de relacionarse con la prevención de enfermedades, tiene un estrecho vínculo con la construcción de un régimen alimentario saludable que favorece el buen estado de salud, mejores condiciones de vida y mayores garantías a la seguridad alimentaria individual y colectiva(16).

Con respecto a las verduras de mayor consumo en el hogar según lo referido por las madres participantes, estas tienen relación con aquellas cuya producción es representativa en Norte de Santander (tomate, zanahoria, habichuela, brócoli, lechuga y repollo) como lo referencia el Ministerio de Agricultura y Desarrollo Rural Regional Norte de Santander(17). Así mismo, el perfil nacional de producción y consumo de frutas y verduras en Colombia, realizado por el Ministerio de Salud y Protección Social y la Organización de las Naciones Unidas para la Alimentación y la Agricultura (FAO), afirma con relación a las verduras, que las más producidas son en su orden el tomate, zanahoria, cebollas de bulbo y de rama y que dada la diversidad de especies, hay otras que sobresalen por sus volúmenes de producción y/o sus áreas de siembra como el repollo, pepino, palmito y la lechuga, considerando a Norte de Santander como uno de los departamentos que sobresale en producción. Además, destaca que las verduras y hortalizas que con mayor frecuencia se consumen corresponden al tomate, cebolla de bulbo, zanahoria, cebolla de rama, arveja verde, habichuela y repollo(16).

Los resultados obtenidos con relación al consumo de verduras en la población infantil difieren de los rangos reportados por las guías alimentarias basadas en alimentos para la población colombiana, en la cual se establece entre uno a dos intercambios de hortalizas y verduras al día para los niños y niñas de 2 - 5 años de edad(15). Dichos resultados son similares a los reportados por Aldarete et al.(5) en los que se refirió que entre los alimentos más rechazados y que menos consumen los niños se encuentran las verduras, específicamente las de hojas verdes, señalando como causas por las madres y docentes, la falta de costumbre de consumo habitual, y por gustos de los padres que son adquiridos por sus hijos; dichos resultados muestran que hay deficientes conocimientos en cuanto a formas de preparación atractivas para incorporar los alimentos beneficiosos, que usualmente son rechazados.

A pesar de las ventajas de producción del país, los colombianos en el 2005 presentaban un bajo consumo de este tipo de alimentos; el cual se vio reflejado en la Encuesta Nacional de la Situación Nutricional en Colombia, en donde el 27,9\% de las personas entre los 2 y los 64 años no incluyeron ninguna verdura. En el 2010, sólo el 16,1\% de las personas ingerían verduras frescas diariamente. La anterior situación es preocupante debido al papel que juegan las frutas y verduras en una dieta saludable y la relación de su ingesta con la prevención de enfermedades no transmisibles relacionadas con la alimentación, lo que implica un esfuerzo por mejorar la situación a corto, mediano y largo plazo, dada la importancia de estos alimentos en la salud pública de los colombianos(18).

Considerando los factores que afectan el consumo de verduras en los niños y niñas, los resultados obtenidos presentan similitud con el estudio realizado por Díaz(19), en el que se refiere que las prácticas culturales se transmiten de una persona a otra; la aceptación de un alimento incrementa en los niños cuando es modelada por adultos, padres, profesores y otros niños, más aún si se tiene en cuenta que la selección de los alimentos durante los primeros años de vida, depende de las actitudes y de las elecciones hechas por los padres y/o cuidadores encargados de la alimentación. Es así como el estudio permite establecer que es notable la influencia de los padres u otros niños frente al consumo, mediante las elecciones que hacen de los alimentos y que modelan el comportamiento; influyen en los sentimientos de aprobación, rechazo o ejemplo de las relaciones con el alimento. De igual manera, en el estudio realizado por Girón et al.(6) se evidenció el bajo consumo de frutas y verduras en los hogares, en cuyo caso las compran, pero los niños no las consumen, específicamente por la falta de conocimiento de los padres con respecto a una buena alimentación, nutrición y los beneficios en el desarrollo cognitivo, físico y el crecimiento de los niños.

Así mismo, en el estudio realizado por Aldalur et al.(20) también se considera que la deficiente ingesta de frutas y verduras en la infancia puede derivar en una generación de adultos con problemas de salud asociados con un bajo consumo de tales alimentos, por lo que este hecho debería ser tomado en consideración desde la perspectiva de la nutrición comunitaria, pues resulta esencial identificar, tratar y corregir la neofobia alimentaria y la alimentación "caprichosa o quisquillosa" con el objetivo de que los 
niños consuman dietas más saludables y coman mayor cantidad de frutas y verduras.

En cuanto a los factores condicionantes para la adquisición de las verduras, los resultados son similares a los obtenidos por Barrero(21), quien identificó que el $70 \%$ de los participantes compran frutas y verduras al menos dos días por semana, refiriendo que la compra diaria de frutas y verduras es un hábito que tiene el 11\% de la población. En este mismo estudio el sitio de compra es la verdulería del barrio, donde se obtiene mejor calidad, precio y más asesoramiento; situación contraria a la referida por las madres participantes quienes manifestaron realizar la compra de verduras en la casa de mercado. Así pues, es importante destacar que la comodidad es una motivación cada vez más importante a la hora de realizar la compra de la alimentación; la calidad de los productos es un factor determinante, incluso por encima de los precios y las ofertas, así como el aspecto del producto.

Referente a las estrategias utilizadas para incentivar el consumo de verduras en los niños(as), Delgado et al.(22) documenta estrategias similares a las obtenidas en este estudio donde existe la relación a la mediación que hace el padre entre el alimento y el consumo por parte del niño, la cual frecuentemente es asociada a una estrategia de presión. Las estrategias que utilizan los padres para favorecer el consumo abarcan estímulos neutrales, razonamientos, premios, chantajes y presión. Sin embargo, los resultados de estas estrategias no siempre son los mejores. Los niños con rechazo alimentario se correlacionan en su mayoría con las técnicas de presión y amenazas de quitar privilegios de alimentos y juegos. En un estudio retrospectivo de adultos, los participantes reportaron que durante la niñez tuvieron experiencias de presión para comer, que los condujeron a un desagrado continuo por esos alimentos(19).

Teniendo en cuenta las estrategias utilizadas por los padres como los castigos y los premios, se refiere que estas no son adecuadas, ya que las técnicas comúnmente utilizadas para incentivar el consumo se realizan mediante la aceptación paulatina y progresiva, con algunas estrategias como la variación en la forma de preparación o inclusión de nuevos ingredientes. Ante esto, se han obtenido resultados donde se evidencia que el ejemplo, sin presión puede favorecer un mejor consumo(19). La presión no es una buena opción para promover el consumo, puesto que esta genera una baja ingesta, predice comentarios negativos acerca de la comida, así como una tendencia hacia el rechazo. Por su parte, los estímulos neutrales, las recompensas y los elogios están más relacionados con la aceptación del alimento. Si bien, los efectos positivos incrementan la satisfacción por un alimento, en este último caso la literatura reporta que ni los premios, ni las felicitaciones promueven una sostenida elección de alimentos saludables; estos no deben tener ningún valor agregado, en forma de recompensa o castigo(23).

De esta manera, Bazzano et al.(24) destacan que la nutrición temprana es de vital importancia para que los niños sobrevivan, crezcan y se conviertan en adultos sanos que puedan llevar vidas gratificantes y contribuir productivamente a sus comunidades, teniendo en cuenta que mejorar la nutrición entre los niños pequeños ha sido reconocido como una prioridad internacional. El impacto de las mejores prácticas dietéticas en la nutrición infantil ha sido ampliamente estudiado y posteriormente promovido por su potencial para mejorar enormemente la salud.

\section{Conclusiones}

Se identificó que las madres participantes tienen conocimientos asertivos sobre la importancia del consumo de verduras en los niños(as) y su relación con el estado de salud, considerando que las de mayor consumo principalmente en el almuerzo y cena en diferentes preparaciones corresponden a las que se producen en la región. Sin embargo, la cantidad consumida es inadecuada puesto que difiere de las recomendaciones establecidas. Se refirieron factores como el lugar, frecuencia de compra y características organolépticas en la selección, como aspectos condicionantes para la adquisición de las verduras, y los elogios, animación y compañía de padres como estrategias positivas utilizadas para motivar el consumo de las verduras, pese a que también utilizan el castigo y el premio.

\section{Agradecimientos}

A los estudiantes de décimo semestre del programa de Nutrición y Dietética que cursaron la Práctica Campos de Acción Profesional, por su participación en la recolección de la información. A las madres de familia del Hogar Infantil que participaron en este estudio.

Conflicto de intereses: Los autores declaran que no existen conflictos de intereses. 


\section{Referencias}

1. Organización Mundial de la Salud. Fomento del consumo mundial de frutas y verduras. Nota descriptiva. 2015. https://www.who.int/dietphysicalactivity/fruit/es/

2. Instituto Colombiano de Bienestar Familiar, Profamilia. Encuesta Nacional de la Situación Nutricional de Colombia, 2005. $\quad$ Ensin. $2005 . \quad 465 \quad$ p. https://www.minsalud.gov.co/sites/rid/Lists/BibliotecaDig ital/RIDE/VS/ED/GCFI/Ensin\%202005.pdf

3. Instituto Colombiano de Bienestar Familiar. Encuesta Nacional de la Situación Nutricional en Colombia 2010. Encuesta Nac la Situac Nutr en Colomb [Internet]. 2010;1(64):325. Available from: https://www.minsalud.gov.co/sites/rid/Lists/BibliotecaDig ital/RIDE/VS/ED/GCFI/Base\%20de\%20datos\%20ENSIN\% 20-\%20Protocolo\%20Ensin\%202010.pdf

4. Coronado. Z. Factores asociados a la desnutrición en niños menores de 5 años. [Internet]. Universidad Rafael Landívar. 2014. Available from: http://biblio3.url.edu.gt/Tesario/2014/09/15/CoronadoZully.pdf

5. Alderete M, Giorgetti AC. Representaciones sociales sobre alimentación saludable en los cuidadores de niños preescolares de Barrio Chingolo, Córdoba, en el año 2017. Universidad Católica de Córdoba - Facultad de Ciencias de la Salud - Licenciatura en Nutrición. 2017. http://pa.bibdigital.uccor.edu.ar/1468/1/AldereteGiorgetti. FR11.pdf

6. Girón N, Plazas KY. Los hábitos alimenticios en la familia y su indicidencia en el desarrollo de los niños y niñas de preescolar del Centro Educativo Rural El Convento del municipio de Trinidad Casanare. Universidad Santo Tomás de Aquino - Facultad de Educación; 2019. https://repository.usta.edu.co/bitstream/handle/11634/1 5918/2019KeniaPlazasNiniGiron.pdf?sequence=1\&isAllowe $\mathrm{d}=\mathrm{y}$

7. Silvera DC, Rodríguez A, Díaz YA, Moya L, Terry B, Rodríguez V. Método cualitativo rápido para evaluar el consumo de alimentos en adolescentes y adultos. Convención Internacional de Salud, Cuba Salud. 2018. http://www.convencionsalud2018.sld.cu/index.php/connv encionsalud/2018/paper/download/1962/791

8. Saldanha D, Colomé CL, Heck T, Nunes N, Vivero V. Grupo focal y análisis de contenido en investigación cualitativa. Index Enferm. 2015;24(1-2):71-5. http://dx.doi.org/10.4321/S1132-12962015000100016

9. Mollericona JY. Pautas metodológicas para la realización de grupos focales. Antecedentes, fundamentos y prácticas. Tinkazos. 2014; 17(36). Disponible en: http://www.scielo.org.bo/scielo.php?script=sci_arttext\&pid =S1990-74512014000200014\&lng=es\&nrm=iso

10. Arias-Gómez J, Villasís-Keever MÁ, Miranda-Novales MG. El protocolo de investigación III: la población de estudio. Rev Alerg México. 2018;63(2):201. http://revistaalergia.mx/ojs/index.php/ram/article/view/ $181 / 309$

11. Attride-Stirling J. Thematic networks: an analytic tool for qualitative research, Qualitative Res. 2001;1(3):385-405. https://utsc.utoronto.ca/ kmacd/IDSC10/Readings/Readi ngs/text\%20analysis/themes.pdf

12. Ministerio de Salud. Resolución No 008430 del 04 de octubre de 1993. Bogotá, Colombia. 1993.
https://www.minsalud.gov.co/sites/rid/Lists/BibliotecaDig ital/RIDE/DE/DIJ/RESOLUCION-8430-DE-1993.PDF

13. Hernández E, Severiche D, Romero-D, López M, Espitia V, Rodríguez A. Promoción de alimentación saludable en hogares comunitarios infantiles del municipio de Sopó (Cundinamarca. Colombia) bajo la estrategia de Atención Primaria en Salud. Rev Científica Salud Uninorte. 2015;31(3). Disponible http://www.redalyc.org/articulo.oa?id=81745378008

14. Alva VC. Conocimientos, percepciones y prácticas alimentarias de madres de escolares con exceso de peso de 6 a 8 años de edad de tres colegios públicos del Cercado de Lima agosto - setiembre 2014. 2017. http://cybertesis.unmsm.edu.pe/handle/cybertesis/6716

15. Instituto Colombiano de Bienestar Familiar. Guías Alimentarias basada en alimentos para la población colombiana mayor de dos años. Manual para facilitadores. Primera edición. 2015. https://www.minsalud.gov.co/sites/rid/Lists/BibliotecaDig ital/RIDE/VS/PP/SNA/guias-alimentarias-basadas-enalimentos.pdf

16. Ministerio de Salud y Protección Social. Lineamiento técnico nacional para la promoción de frutas y verduras. Estrategias para el aprovisionamiento, manejo, expendio y promoción del consumo de frutas y verduras. Bogotá D.C. 2013. http://ecos-redenutri.bvs.br/tikidownload_file.php?fileId=1647.

17. Ministerio de Agricultura y Desarrollo Rural. Anuario Agrícola regional Norte de Santander, cultivos producción en toneladas (T). 2016.

18. Ministerio de Salud y Protección Social. Perfil nacional de consumo de frutas y verduras. Bogotá D.C. 2013. https://www.minsalud.gov.co/sites/rid/Lists/BibliotecaDig ital/RIDE/VS/PP/SNA/perfil-nacional-consumo-frutas-yverduras-colombia-2013.pdf

19. Díaz M. Factores influyentes en el comportamiento alimentario infantil. Rev Fac Med. 2014;62(2):237-245. http://www.scielo.org.co/pdf/rfmun/v62n2/v62n2a10.pdf

20. Aldalur EM, Mateo CM, Lasa NB. Neofobia y otros trastornos restrictivos alimentarios en la infancia y consumo de frutas y verduras: revisión. Rev Española Nutr Comunitaria. 2014;20(4):150-7. DOI:10.14642/RENC.2014.20.4.5029

21. Barrero L. Estudio sobre hábitos de consumo de frutas y verduras de los consumidores cordobeses. Documento de Trabajo.

2012 https://desarrolloterritorial.adec.org.ar/horticola/images/ habitos-de-consumo-de-frutas-y-verduras.pdf

22. Delgado-Pérez D, Liria-Dominguez R. Estrategias usadas para alimentar a niños preescolares por madres de una zona urbano marginal de Lima, Perú. Rev Peru Med Exp Salud Publica. 2016;33(3):507. https://doi.org/10.17843/rpmesp.2016.333.2295

23. Uceda EM. Influencia del comportamiento en las conductas en alimentación de niños de educación infantil [Internet]. 2015. Available from: http://digibug.ugr.es/bitstream/handle/10481/40949/Ma ya_Uceda_Elena.pdf;jsessionid=E00D6ADA00DA697E6C4CC 2FC3840075F?sequence $=1$

24. Bazzano AN, Potts KS. Qualitative Studies of Infant and Young Child Feeding in Lower-Income Countries: A Systematic Review and Synthesis of Dietary Patterns. Nutrients. 2017;18(9). DOI:10.3390/nu9101140 